Volume 8

Issue 4 - Cancer Screening

Article 12

10-18-2021

\title{
Conference Proceedings: Select Abstracts Presented at 2021 Advocate Aurora Health Scientific Day
}

Follow this and additional works at: https://aah.org/jpcrr

Part of the Analytical, Diagnostic and Therapeutic Techniques and Equipment Commons, Behavioral Medicine Commons, Cardiology Commons, Critical Care Commons, Diseases Commons, Family Medicine Commons, Infectious Disease Commons, Internal Medicine Commons, Medical Education Commons, Neurology Commons, Neurosurgery Commons, Obstetrics and Gynecology Commons, Oncology Commons, Palliative Care Commons, Pediatrics Commons, Pharmacy and Pharmaceutical Sciences Commons, Primary Care Commons, Public Health Commons, Pulmonology Commons, Substance Abuse and Addiction Commons, and the Translational Medical Research Commons

\section{Recommended Citation}

Conference proceedings: select abstracts presented at 2021 Advocate Aurora Health Scientific Day. J Patient Cent Res Rev. 2021;8:363-75. doi: 10.17294/2330-0698.1920

Published quarterly by Midwest-based health system Advocate Aurora Health and indexed in PubMed Central, the Journal of Patient-Centered Research and Reviews (JPCRR) is an open access, peer-reviewed medical journal focused on disseminating scholarly works devoted to improving patient-centered care practices, health outcomes, and the patient experience. 


\section{Conference Proceedings: Select Abstracts Presented at 2021 Advocate Aurora Health Scientific Day}

Abstracts published within this supplement were presented at the 47th annual Advocate Aurora Health Scientific Day, held virtually on May 26, 2021. This research symposium provides a forum for disseminating results from studies conducted by faculty, fellows, residents, and other allied health professionals associated with Midwest-based health system Advocate Aurora Health, which publishes the Journal of Patient-Centered Research and Reviews.

\section{ORAL PRESENTATIONS}

Clinical Outcomes of Critically III Patients With COVID-19: A Single-Center Cohort Study

Kenneth W. Dodd, Esmael Mayar, Brianna Miner, Gisele Papo, Nathan Schoenrade, Paige Bird, David Barounis, Katharine Burns, Nicholas Goodmanson, Varsha P. Gharpure

Emergency Medicine, Advocate Christ Medical Center; Internal Medicine, Advocate Christ Medical Center; Advocate Children's Hospital

Background: A global pandemic due to COVID-19 was declared in March 2020. Cook County, Illinois, was an early epicenter of the outbreak. Severe COVID-19 may result in respiratory failure requiring mechanical ventilation or multisystem organ failure requiring various organ support therapies.

Purpose: To describe the outcomes of critically ill patients with COVID-19.

Methods: This prospective cohort study analyzed adult patients admitted to Advocate Christ Medical Center (Oak Lawn, IL) between March 1, 2020, and May 1, 2020, who were treated in a medical intensive care unit for laboratory-confirmed COVID-19. Primary outcome was in-hospital mortality. Secondary outcomes were discharge location and length of stay. Results are stratified by age and need for organ support (mechanical ventilation, renal replacement therapy, and extracorporeal membrane oxygenation [ECMO]). The primary analysis is descriptive in nature.

Results: In total, 218 patients were admitted during the study period. Mean (standard deviation) age was 65.2 (13.4) years; 129 patients (59.2\% were male, 91 (42\%) were White, 82 (38\%) were Black, and $34(16 \%)$ were Hispanic. Incidence of organ support modalities included $79(36 \%)$ mechanical ventilation, $34(16 \%)$ renal replacement therapy, and 2 (1\%) ECMO. Overall in-hospital mortality was $29 \%(n=80)$. For patients requiring organ support, mortality was 33 of 79 (42\%) for mechanical ventilation, 25 of 34 (75\%) for renal replacement therapy, and 2 of $6(33 \%)$ for ECMO. Rates of discharge home were 63 of $125(50 \%)$ for patients who did not require organ support therapies, 24 of 79 (30\%) for mechanical ventilation, 3 of $34(9 \%)$ for renal replacement therapy, and 2 of $6(33 \%)$ for ECMO. For patients requiring mechanical ventilation, median (interquartile range) hospital length of stay was 17.3 (11.3-27.5) days. In patients older than age 74 who required mechanical ventilation, in-hospital mortality was 11 of $16(69 \%)$ and $0 \%$ were discharged to home.

Conclusion: Need for organ support modalities and increasing age were associated with higher mortality and lower rate of discharge to home during the early experience with COVID-19 at Advocate Christ Medical Center. This study provides important baseline data for clinical planning and future research.
Detecting Cardiac Disease Using Artificial Intelligence With 12-Lead Electrocardiographic Data

Christopher R. Beal, M. Fuad Jan, Arshad Jahangir, A. Jamil Tajik

Advocate Aurora Research Institute; Aurora Cardiovascular Services, Aurora St. Luke's Medical Center

Background: Cardiac diseases such as hypertrophic cardiomyopathy (HCM) and aortic valve stenosis (AVS) affect the heart's main blood-pumping chambers and can result in adverse outcomes like sudden cardiac death. The echocardiogram is the gold standard to diagnose these diseases. Equipment and expertise to perform this procedure can be costly in some health systems; 12-lead electrocardiograms (ECG) are lower-cost tools to establish baseline cardiac function. However, these diseases present similarly on ECGs, and distinguishing them is a clinical challenge.

Purpose: To confirm similar disease presentation, to detect disease presence, and to identify features most indicative to detect disease, using artificial intelligence.

Methods: A total of 16,781 ECGs were processed from 1461 adult patients diagnosed with one disease after undergoing a previous echocardiogram. A multiclass study design was used to detect disease presence; 912 features were extracted from raw ECG signals in the MUSE database. Training data consisted of $75 \%$ of the observations $(n=1096)$, and testing data consisted of the remaining $25 \%$ of the observations $(n=365)$. Feature selection with the SelectKBest method reduced 912 features to a subset of 50 important features. Principal component analysis was used to visualize how each disease presented from the others. Multilayer perceptron and linear discriminant analysis models classified diseases and were compared in terms of accuracy, positive predictive value, and area under the curve.

Results: Principal component analysis showed one overlapping cluster of 6 diseases, indicating data patterns for each disease are not wellseparated and confirming the challenge of separating scans. Multilayer perceptron most accurately detected Fabry's disease (83.6\%); linear discriminant analysis most accurately detected amyloidosis (75.4\%). From the 50 important features, feature values and lead numbers characterized the diseases. S-wave features characterized amyloidosis, HCM, and AVS (21, 17, and 17 occurrences, respectively); T-wave features characterized Fabry's disease (14 occurrences); R-wave features characterized left ventricular hypertrophy and sarcoidosis (13 occurrences). Lead V6 characterized amyloidosis, AVS, HCM, and sarcoidosis $(8,11,9$, and 8 occurrences, respectively); Lead I characterized left ventricular hypertrophy ( 8 occurrences); Lead III characterized Fabry's disease (13 occurrences).

Conclusion: The choice of classification model depends on the chosen disease, and artificial intelligence is a useful tool in clinical practice in conjunction with physicians' trained expertise. 


\section{Zika Virus Demonstrates Oncolytic Activity in a Patient- Derived Glioblastoma Xenograft Model in Mice}

Parvez Akhtar, Sam D. Zwernik, Deborah L. Donohoe, Dmitry Bosenko, Catherine Warner, Richard A. Rovin

\section{Advocate Aurora Research Institute; Neurosurgery, Aurora Neurosciences Innovation Institute}

Background: Glioblastoma is a malignant primary brain cancer. The poor median survival rate of 15 months for patients with glioblastoma has not budged in the past 15 years, when the current standard treatment was first approved. There is no standard-ofcare chemotherapy for recurrent glioblastoma. We hypothesized that Zika virus (ZIKV) can be purposed as an oncolytic virus for glioblastoma. In order to test this hypothesis, we have used patientderived xenograft mouse model and investigated the oncolytic potential of ZIKV.

Purpose: Preliminary in vitro data from our lab confirms that ZIKV has tropism for commercial and patient-derived glioblastoma cell lines through the AXL receptor, and the subsequent productive infection is cytotoxic. Given this information, we wanted to determine if ZIKV can work as an oncolytic virus against glioblastoma in mouse models.

Methods: All procedures were approved by the Institutional Animal Care and Use Committee. We used 6-8-week-old Fox N1 Nude homozygous female mice. There were 4 experimental arms: 2 patient-derived cell lines, each with a ZIKV-treated group and a control group. The number of animals per experimental arm was 12. Animals received subcutaneous flank injections of patient-derived glioblastoma 8049 or 8049 AXLKO cells $(2 \times 106)$ in $100 \mu \mathrm{l}$ matrigel under isoflurane anesthesia. Animals were observed daily, and weights and tumor measures were done 3 times per week. When tumor size reached $150 \mathrm{~mm}^{3}$, mice received intratumoral injection of $2.5 \times 106$ ZIKV particles; control group received intratumoral saline. At euthanasia, tissues were harvested for immunohistochemistry and qRT-PCR was used to determine ZIKV distribution.

Results: After 2-4 weeks of viral particle administration, striking results were observed. ZIKV induced complete tumor remission in 22 of 24 animals (8049: 11 of 12; 8049 AXLKO: 11 of 12). There was no tumor remission in 8049 and 8049 AXLKO control animals. Overall survival of 8049 and 8049 AXLKO tumor-bearing mice was significantly improved $(\mathrm{P}=0.001)$ by ZIKV treatment: median survival of ZIKV-treated animals was 124 days for 8049 and 125 days for 8049 AXLKO, which compared to 42 days for 8049 and 46 days for 8049 AXLKO in control animals. Among ZIKV-treated mice, there were 2 recurrences -1 in the 8049 tumor (24 days after significant tumor remission) and 18049 AXLKO tumor (7 days after significant tumor remission). We found a significant amount of virus particles in ZIKV-infected tumors using qRTPCR. Histologic analysis confirmed necrotic tumor foci and ZIKVpositive tumor cells in treated animals.

Conclusion: ZIKV could be a potential oncolytic agent for glioblastoma therapy.
Treatment Outcomes and Program Transition Rates Across Substance Use Treatment Programs

Mindy R. Waite, Kayla Heslin, Jonathan Cook, Michelle Simpson

Aurora Behavioral Health Services, Advocate Aurora Research Institute; Aurora UW Medical Group; Center for Urban Population Health; Ed Howe Center for Research, Aurora Sinai Medical Center

Background: Patients with substance use disorder represent a diverse group of individuals with unique histories, demographics, substance use patterns, and treatment-related behaviors. As a result, several levels of treatment programs exist, including inpatient, residential, partial hospitalization (PHP), intensive outpatient (IOP), and outpatient. Program level is based on current clinical needs, and best practice is to successfully complete a program and transition to the next in a step-down manner. In contrast, patients who discharge against medical advice, have lower lengths of stay, or fail to transition are thought to have worse outcomes. However, actual patient behaviors and their outcomes within and across substance use treatment programs are not well understood.

Purpose: To describe the outcomes and transition rates of patients across substance use treatment programs.

Methods: Medical records were used to collect data from substance use disorder treatment programs at a Midwest-based psychiatric hospital between January 1, 2017, and December 31, 2019. Data included treatment history, reason for discharge, admissions, latency to program transitions, and location. Basic descriptive and comparative statistics were conducted.

Results: Programs differed in rates of discharge against medical advice. PHP had the highest rate of discharge against medical advice, whereas residential treatment had the lowest. In most programs, patients who discharged against medical advice had similar rates of successful program transitions compared to those who completed the program. Inpatients had higher rates of 6-month readmissions than any other treatment program. Whereas lower acuity treatment programs (residential, PHP, IOP) had decreased inpatient readmissions following program discharge, inpatients had higher inpatient admission rates postdischarge compared to before admission. Consistent with this, programs had unique transition patterns, where inpatients were most likely to readmit as inpatients, residential patients were most likely to step down to PHP, PHP patients typically stepped down to IOP, and IOP discharges transitioned across several programs. However, a large subset of patients did not successfully transition to any program. Insurance type was a factor in transitioning to residential treatment.

Conclusion: Treatment-related behaviors and outcomes of patients were unique across programs, potentially reflecting differences in treatment intensities and patient acuities. Results highlight opportunities for reducing readmissions and increasing successful transitions. 


\section{Anticoagulation for COVID-19-Associated Coagulopathy: Experience of a Single Community Hospital}

Dana Mueller, Tyler Cooper, Lauren Sesemann, Lyndsey Turbow, Nancy Guirguis, Marc McDowell, Poorvi Shah, Bassem Chaar, Gautam Raju Mehta, Kenneth W. Dodd

Internal Medicine, Advocate Christ Medical Center; Pharmacy, Advocate Christ Medical Center, Hematology and Oncology, Advocate Christ Medical Center; Critical Care Medicine, Advocate Christ Medical Center

Background: Thrombotic events despite prophylactic anticoagulation are a well-recognized complication in patients diagnosed with severe COVID-19. The appropriate dosage and indications for anticoagulation remains controversial.

Purpose: To describe the general outcomes of critically ill COVID-19 patients receiving therapeutic anticoagulation guided by our expert-driven protocol.

Methods: This retrospective cohort study includes patients admitted to the intensive care unit between March 3, 2020, and April 12, 2020, at Advocate Christ Medical Center (Oak Lawn, IL). Patients taking oral anticoagulants prior to arrival and those started on therapeutic anticoagulation to prevent extracorporeal membrane oxygenation (ECMO) circuit thromboses were excluded. The initiation and dosing of anticoagulation was at the discretion of the treating clinicians. Informal, institutional indications for initiation of therapeutic anticoagulation were: D-dimer of $>6$ times the upper limit of normal (ULN), sepsis-induced coagulopathy of $\geq 4$, and clinical evidence of thrombosis. Demographic, laboratory, and clinical outcomes data were collected. Thrombotic and bleeding events were adjudicated by 2 trained clinicians or pharmacists. Results are descriptive.

Results: Of 145 subjects identified, 67 (46\%) received therapeutic anticoagulation. Median (interquartile range [IQR]) age was 61 (56-71) years, median (IQR) body mass index was 32.8 (29-36.7) $\mathrm{kg} / \mathrm{m}^{2}, 42(62 \%)$ were male, and $44(66 \%)$ were non-White. The most common comorbidities were hypertension $(n=50,75 \%)$ and obesity $(n=46,69 \%)$. The initial therapeutic anticoagulation method was unfractionated heparin infusion in $63(94 \%)$ and subcutaneous enoxaparin in $4(6 \%)$. The most common indication was D-dimer of $>6 \times \mathrm{ULN}$ in $58(87 \%)$, while $4(6 \%)$ were started on anticoagulation for thrombotic events alone. Median (IQR) time to initiation was 5 (1.5-8) days; 43 (64\%) required invasive mechanical ventilation at the time of initial therapeutic anticoagulation. At follow-up, 44 (65\%) subjects were discharged alive, and 23 (34\%) had expired. Four $(6 \%)$ were diagnosed with 5 new thrombotic complications: 3 (4.5\%) deep vein thromboses, $1(1.5 \%)$ pulmonary embolism with hemodynamic compromise, and $1(1.5 \%)$ ischemic cerebrovascular accident. Two (3\%) patients experienced clinically overt gastrointestinal bleeding accompanied by a decrease in hemoglobin of at least $2 \mathrm{~g} / \mathrm{dL}$. No other fatal or major bleeding complications reported. Minor bleeding events were identified in $12(18 \%)$ subjects.

Conclusion: In this study, critically ill adults on therapeutic anticoagulation had a low incidence of new thrombotic complications and low incidence of major bleeding.
Effects of Therapeutic Anticoagulation for SARS-CoV-2 Coagulopathy on Markers of Inflammation and Mortality

Lyndsey Turbow, Nancy Guirguis, Praveena Tathineni, Dana Mueller, Tyler Cooper, Daniel Thompson, Nicholas Goodmanson, John Weissert, Kourosh Ravvaz, Kenneth W. Dodd

Internal Medicine, Advocate Christ Medical Center; Pulmonology and Critical Care, Advocate Christ Medical Center; Advocate Aurora Research Institute; Emergency Medicine, Advocate Christ Medical Center

Background: Practitioners have used therapeutic anticoagulation to prevent the thrombotic complications of the severe acute respiratory coronavirus 2 (SARS-CoV-2) responsible for the COVID-19 pandemic. The effect of such an approach on serum inflammatory markers, and factors associated with survival while on therapeutic anticoagulation, are not known.

Purpose: This study evaluated the effect of therapeutic anticoagulation on C-reactive protein (CRP), lactate dehydrogenase (LDH) ferritin, and D-dimer and also analyzed the association of several factors with mortality.

Methods: This retrospective cohort study used data collected from patients diagnosed with SARS-CoV-2 and receiving therapeutic anticoagulation in the intensive care unit of a tertiary care center between March 3, 2020, and April 12, 2020. Indications for initiation of therapeutic anticoagulation included D-dimer of $>6$ times the upper limit of normal (ULN), sepsisinduced coagulopathy score of $\geq 4$, or evidence of thrombotic disease. Data included initial serum markers and repeat values taken at discreet intervals, along with clinical features and mortality. Comparisons were made using 2-sided Mann-Whitney $\mathrm{U}$ test. Multivariate analyses for predicting death were calculated using Cox proportional hazards models.

Results: Of 145 subjects, $67 \quad(46 \%)$ received therapeutic anticoagulation: $58(87 \%)$ for COVID-19 coagulopathy and 12 (18\%) for thrombotic events. Initial median (interquartile range) serum values were as follows: CRP of 14 (9-21) mg/dL, D-dimer of $8.1(4.3-18.6) \mathrm{mg} / \mathrm{L}$, ferritin of $1614(723-2526) \mathrm{ng} / \mathrm{mL}$, and LDH of 445 (356-629) units/L. Five days after initiation of anticoagulation, patients had a decrease in all measured median serum values: CRP of 6.8 (2.5-9), LDH of 385 (297-472), D-dimer of 4.1 (2.3-7.9), and ferritin of 975 (646-1483); $\mathrm{P}<0.05$ for all). At the time of follow-up, 23 (34\%) patients had been discharged alive, $20(30 \%)$ patients had expired, and $24(36 \%)$ patients remained in-hospital. Mortality was directly associated with age (hazard ratio [HR]: 9.05, 95\% CI: $1.71-46$; $\mathrm{P}<0.01$ ) and postanticoagulation D-dimer level (HR: 9.78, 95\% CI: 1.81-52.9; $\mathrm{P}<0.01$ ) and inversely associated with obesity (HR: $0.27,95 \%$ CI: $0.80-0.93 ; \mathrm{P}<0.05$ for all).

Conclusion: In this patient population, therapeutic anticoagulation was associated with a decrease in inflammatory markers, suggesting efficacy of treatment. Anticoagulation may have a particularly important role in obese patients, as obesity was associated with higher survival in patients placed on anticoagulation. This study provides important data for larger, prospective trials. 


\section{Advancing Advance Directives in an Internal Medicine Residency Clinic}

Tanya Shah, Ramandeep Dhaliwal, Zeba Shethwala, Henok Hardilo, Jasmine Webster, David Hamel, Ankoor Biswas, Deborah Simpson

Internal Medicine, Aurora Sinai Medical Center; Aurora UW Medical Group, Family Medicine and Academic Affairs, Aurora Sinai Medical Center

Background: Research shows $89 \%$ of patients prefer advance directive (AD) conversations be initiated in the outpatient setting, ideally with their primary care doctor. Only $47 \%$ of patients in our resident clinics who are older than 65 years of age have completed advance directives documentation on file.

Purpose: To increase AD completion numbers for $>65$-year-old patients in an internal medicine residency clinic by $12 \%$.

Methods: We developed a new standardized workflow for all clinic patients who are $>65$ years of age to ensure they were receiving appropriate goals-of-care counseling. During the pandemic, the workflow was adapted to include virtual $\mathrm{AD}$ completion utilizing a new organizationally approved online platform and incentivizing clinic teams for AD-specific appointments. The new workflows were implemented at 1 of 2 internal medicine residency clinics, with the other used as a control site. Residents completed a series of educational lectures and simulation exercises on how to engage in advance care planning discussions in the outpatient setting. Metrics included health system-provided percentages of $\mathrm{AD}$ completion rates, with overall clinic quality improvement scores and Clinical Learning Environment Quick Surveys (CLEQS) used as balancing measures. Results: Between January 2020 and December 2020, our clinic AD completion improved $1 \%$ at the intervention site. Postvirtual $\mathrm{AD}$ implementation, $\mathrm{AD}$ intervention site completion rate increased another $1 \%$ in January 2021. This marks an overall 4\% $\mathrm{AD}$ intervention site improvement compared to the control site at which $\mathrm{AD}$ completion rate decreased by $2 \%$ during the same period. There were no unexpected changes in our overall quality improvement scores and a pre/post-project implementation improvement, as measured by CLEQS, in teamwork.

Conclusion: Planning for future health care needs has multiple benefits for older adults, their loved ones, and the entire health system. Improving provider comfort with and destigmatizing advance planning conversations is an essential step in promoting the completion of AD documentation. As seen with our January 2021 data, better coordination with clinic social work staff through clear workflows and availability and utilization of online AD completion platforms is critical in progressing toward goal achievement. Moving forward, continuing to integrate new virtual AD technology into clinic operations and provider training appears to facilitate $\mathrm{AD}$ document completion in a more timely and efficient manner.

\section{Opioid Prescribing Practices Following Otologic and Neurotologic Surgery}

\section{Sarah J. Riutta, Aaron G. Benson}

Advocate Aurora Research Institute; Otolaryngology Head and Neck Surgery, Aurora Sinai Medical Center

Background: Surgeons accounted for nearly 37\% of opioid prescriptions dispensed in the United States between 2007 and
2012. Prescribing opioids for postoperative pain is routine, but there is little consensus on the appropriate amount to prescribe. Recent publications have found the number of pills prescribed by otolaryngologists following ear, nose, and throat procedures varies widely, and patients typically do not consume the entirety of their prescription. Given the variability in reported prescribing practices and limited data available specific to otologic and neurotologic procedures, there is a need to further examine opioid prescribing practices.

Purpose: To provide insight into opioid prescribing practices following otologic and neurotologic procedures at Aurora Health Care.

Methods: Medical records of patients who underwent an otologic or neurotologic procedure at Aurora Health Care between October 1, 2018, and August 24, 2020, were examined for inclusion. Records were included if patients were not chronic opioid users prior to surgery and were not admitted to the hospital within 2 weeks of surgery.

Results: A total of 230 patient records met eligibility criteria. All but 4 patients received a postoperative opioid prescription. Nearly $90 \%$ of prescriptions were written for 30 tablets of 5-325 mg hydrocodone-acetaminophen. Refills were provided to $16 \%$ of patients. The cohort or patients that received refills was significantly younger, reported higher pain scores during post-anesthesia care unit (PACU) recovery, and received significantly more intravenous fentanyl during the perioperative period compared to the patient cohort that did not receive a refill. Patients were more likely to receive a refill if they had preoperative otalgia or received any opioid analgesic during PACU recovery. There was no significant effect of sex, race, ethnicity, body mass index, smoking status, or recent preoperative narcotic exposure on whether a patient received a refill of opioid pain medication.

Conclusion: Younger age, preoperative otalgia, high inpatient opioid dose requirements, and high inpatient pain scores were associated with refills of opioid prescriptions following otologic and neurotologic surgery. Further research is needed to clearly define factors otologic surgeons should consider for tailoring postoperative opioid prescriptions to individual patient needs.

\section{National Cancer Database Analysis of Outcomes in Patients Diagnosed With Glioblastoma}

\section{Maharaj Singh, Parvez Akhtar, Richard A. Rovin}

\section{Advocate Aurora Research Institute; Neurosurgery, Aurora Neurosciences Innovation Institute}

Background: Glioblastoma is one of the aggressive types of cancer that can occur in the brain or in spinal cord. There has been progress in understanding in recent years; however, prognoses are still very poor. Treatment of glioblastoma requires a multidisciplinary approach based on understanding the pathophysiology of the disease, which can eventually lead to a better prognosis for patients. Purpose: To describe the demographics for the patients diagnosed with glioblastoma and evaluate association of patient characteristics, socioeconomic status, and treatment with overall survival at 1,2 , and 5 years of diagnosis.

Methods: We analyzed the National Cancer Database, which provides broad and detailed information on demographic characteristics, treatment modalities, and survival outcome for patients diagnosed with glioblastoma. Descriptive statistics, Kaplan-Meier curve, and Cox proportional hazards regression were used to analyze the data. For all statistical analyses, SAS 9.4 software (SAS Institute Inc.) was used. 
Results: A total of 124,282 patients were identified with diagnosis of glioblastoma from 2004 through 2016. The study population included patients with ICD-O-3 histology codes 9440, 9441, and 9442 and tumor sites for central nervous system and brain (C71.0C71.9 and C72.0-C72.3). Data with missing vital status (dead, alive) were excluded from the analysis. Population was $57.4 \%$ male, $87 \%$ non-Hispanic White (NHW), 5.5\% non-Hispanic Black (NHB), 5\% Hispanic, and rest were from the "Other" race category. Adjusted hazard ratio for mortality was significantly higher for males (1.06) and for NHW (1.3 compared to Hispanics, 1.2 compared to NHB, and 1.3 compared to "Others"). Similarly, hazard ratios were higher for low-income group (1.03), uninsured (1.06), and for patients with higher comorbidity scores (1.2-1.4). The patients with treatment such as surgery $(0.57)$, radiation $(0.69)$, immunotherapy (0.83), or chemotherapy (0.64) had lower hazard ratio for mortality. Every 10-year increase in age also was found to cause a 1.27 times increase in mortality. Respective overall survival percentages at $1,2,3$, and 5 years were only $42 \%, 19 \%$, $11 \%$, and $6 \%$ of the patients diagnosed with glioblastoma.

Conclusion: Female, Hispanic and Asian/Pacific Islanders, and younger patients had better survival. Patients who underwent treatments such as surgery, chemotherapy, immunotherapy, hormonal therapy, and radiation had better survival probability.

\section{Risk Factors for Developing Unpleasant Paresthesia After Implantation of Permanent Spinal Cord Stimulators}

\author{
Nebojsa Nick Knezevic, Tabish Aijaz, Alvaro Camacho- \\ Ortega, Kenneth D. Candido
}

\section{Anesthesiology, Advocate Illinois Masonic Medical Center}

Background: Spinal cord stimulators (SCS) have become a promising option to treat chronic pain syndromes; nevertheless, there are challenges that might compromise the effectiveness and lead to higher health care costs. Unpleasant paresthesia/dysesthesia is one of these challenges.

Purpose: To determine the incidence of unpleasant paresthesia after implantation of permanent SCS and find the risk factors for its development.

Methods: After institutional review board approval, a retrospective analysis was conducted of prospectively collected data of patients that had permanent percutaneous SCS implanted. We included patients $>18$ years of age with chronic intractable nonmalignant pain of the trunk and/or limbs who had SCS implanted for at least 2 years. We used SPSS 22.0 software (IBM Corp.) to perform statistical analysis.

Results: A total of 104 patients had at least 24 months of followup after implantation of permanent SCS. Mean age of patients was 56.99 years, and the average body mass index was 29.7. Mean pain score before implantation, measured via numeric rating scale (NRS), was 8.05 , which was reduced to 5.6 after 24 months. Excluding the need for routine reprogramming, $45 \%$ of the complication events presented within the first 12 months of follow-up $(\mathrm{P}=0.022)$. The most common complication in our study sample was the development of unpleasant paresthesia/dysesthesia, which was reported by $27 \%$ of participants. There was no association between paresthesia and age, gender, or body mass index. We found a statistically significant correlation between current tobacco use and cases of uncomfortable paresthesia and/or dysesthesia in patients that received the permanent $\mathrm{SCS}(\mathrm{P}=0.001)$, and $38 \%$ of the cases required or requested explantation of the device $(\mathrm{P}=0.028)$. During the follow-up period, active tobacco users and former tobacco users had a lower-than-average mean NRS reduction of $2.74 \pm 2.30$ and $2.90 \pm 3.90$, respectively, when compared to a $3.33 \pm 2.67$ mean NRS reduction in never tobacco users; however, no statistical significance was observed between the groups $\left(\mathrm{F}_{(<1)} \mathrm{P}=0.60\right)$.

Conclusion: Unpleasant paresthesia seems to be more common than originally thought, with an unclear cause. According to our study findings, longstanding tobacco users being treated with SCS are more prone to develop unpleasant paresthesia/dysesthesia when compared with non- and former tobacco users.

\section{Clinical Characteristics and Outcome of Children Hospitalized With SARS-CoV-2-Related IIIness: A Single- Center Descriptive Cohort Study}

\section{Varsha P. Gharpure, Kenneth W. Dodd}

\section{Advocate Children's Hospital; Emergency Medicine, Advocate Christ Medical Center}

Background: Pattern of presentation and hospital course of children affected with SARS-COV-2 infection is distinct from adults.

Purpose: To describe characteristics and outcomes of children affected by SARS-CoV-2-related illness presenting to two campuses of a Midwestern community-based children's hospital. Methods: Data of symptomatic hospitalized children with laboratory-confirmed SARS-CoV-2 infection or epidemiologically related multisystem inflammatory syndrome in children (MIS-C) as defined by Centers for Disease Control and Prevention (CDC) - who were reported to the Society of Critical Care Medicine's VIRUS COVID-19 registry from two campuses of Advocate Children's Hospital (Park Ridge, IL) between March 1, 2020, and March 31, 2021, were retrospectively analyzed. Primary outcome measure was in-hospital mortality; secondary outcome measures were hospital length of stay, need for admission to the intensive care unit, and organ support. Data are reported as percentage, number, or median (interquartile range) and analyzed with Fisher's exact, chi-squared or Mann-Whitney U tests as appropriate.

Results: A total of 186 children were admitted during the study period (median age: 5.7 [1.7-12.2] years; male gender: 60\%; Black race: $17 \%$; Hispanic ethnicity: $44 \%$ ), and 61 met the CDC definition of MIS-C. SARS-COV-2 PCR assay was positive in 152, while immunoglobulin $\mathrm{G}$ antibody was positive in 59. Overall, 180 patients were discharged from the hospital, 5 were transferred to another facility, and 1 patient on chronic noninvasive home ventilation died; 133 were reported to be alive at day 28 , and the rest were lost to follow-up. Median duration of hospitalization was 3.6 (1.9-5.8) days; 89 children needed intensive care (median duration: 2.5 [1.3-50] days). Modalities of organ support included invasive mechanical ventilation $(n=25$, median of 1.7 [0.1-90] days), noninvasive ventilation $(n=11)$, high-flow nasal canula $(n=36)$, nitric oxide $(n=7)$, and extracorporeal membrane oxygenation $(n=3)$. Compared to those without MIS-C, children presenting with MIS-C were older (8 [4.3-10.9] years vs 4.3 [0.513.45 ] years; $\mathrm{P}=0.01)$, less likely to have comorbidities $(\mathrm{P}=0.002)$, and more likely to need intensive care $(\mathrm{P}<0.001)$.

Conclusion: Children demonstrate two distinct phenotypic presentations related to SARS-CoV-2 infection, a typical COVID-19 type and a distinct multisystem inflammatory syndrome. Early identification of risk factors is essential for appropriate intensive care utilization and management decisions. 


\section{POSTER PRESENTATIONS}

Comparison of Treprostinil and Phosphodiesterase Type 5 Inhibitor for the Treatment of Systemic Sclerosis: A Retrospective Study

Sheldon R. Garrison, Angela A. Navarrete-Opazo, Dianne L. Zwicke, Alex M. Maley, Maharaj Singh, Sara Paulus

Advocate Aurora Research Institute; Transplant Cardiology, Aurora St. Luke's Medical Center; Aurora Dermatology, Advocate Aurora Health

Background: Systemic sclerosis is a rare connective tissue disease affecting approximately 100,000 people in the United States. Onset is typically between 30 and 60 years of age, with gradual progression over 11-22 years. Systemic sclerosis is characterized by diffuse fibrosis of the skin and internal organs, vascular insufficiency, and autoimmune dysregulation. Nearly half of systemic sclerosis patients develop digital ulcers, which are profoundly painful, slow to heal, and may progress to osteomyelitis or even amputation. Despite the high unmet need, only one drug has been approved by the U.S. Food and Drug Administration for the treatment of systemic sclerosis-associated interstitial lung disease. Thus, there is an immediate need to identify and test therapeutics for the treatment of digital ulcers in systemic sclerosis.

Purpose: To compare the efficacy of treprostinil and phosphodiesterase type 5 (PDE5) inhibitor for the treatment of cutaneous systemic sclerosis.

Methods: A retrospective chart review of adult patients with a clinical diagnosis of scleroderma or systemic sclerosis within Advocate Aurora Health was performed. Patients received either treprostinil, PDE5 inhibitor, or nonactive treatment (No $\mathrm{Tx}$ ), beyond antibiotic and pain medication, for digital ulcers between January 2011 and March 2020. Treatment efficacy was determined by the rate of digital healing and amputations in each group. One-way ANOVA was used for comparisons of the groups. For all statistical tests, an alpha of 0.05 was used. All statistical analysis was done using Prism (GraphPad Software) and SAS 9.4 (SAS Institute Inc.) software. This study was approved by the institutional review board.

Results: Digital ulcers were observed in 12 of 31 (38\%) patients from the treprostinil-treated group, 7 of 14 (50\%) PDE5-treated patients, and 8 of $13(62 \%)$ No Tx patients. Treprostinil was successfully used to reduce ulcer burden and avoid amputation in $100 \%$ of patients (12 of 12) with digital ulcers. All digital ulcers in treprostinil-treated patients with systemic sclerosis fully resolved, and no new lesions were observed during treatment. Treatment was well tolerated with no major complications. In the PDE5-inhibitor cohort, digital ulcers healed in only $14 \%$ of patients ( 1 of 7$) ; 0 \%$ of patients ( 0 of 8$)$ improved in the No Tx group $(\mathrm{P}<0.001)$.

Conclusion: The findings support the potential efficacy of treprostinil as a therapy for the treatment of digital ulcers in systemic sclerosis.
Prognostic Implications of Vitamin D in Patients With a Hematologic Malignancy at a Large Health System in Wisconsin

Daniel M. Mundt, Marianne Klumph, Kayla Heslin, Michael A. Thompson

Internal Medicine, Aurora Sinai Medical Center; Aurora UW Medical Group; Center for Urban Population Health; Oncology Precision Medicine Program, Advocate Aurora Research Institute

Background: Vitamin D deficiency (VDD) is common in the United States $(\sim 10 \%)$. Epidemiological studies correlate with increased risks for cancer, infections, autoimmune, and cardiovascular diseases when vitamin D levels are less than $20 \mathrm{ng} / \mathrm{mL}$. Single-center studies have shown that VDD at diagnosis may be associated with poorer prognosis in various cancers, including hematologic malignancies for which therapy response may be modulated.

Purpose: To assess the incidence and prevalence of VDD among patients with hematologic malignancies in a large community health system.

Methods: Patients with hematologic malignancies from January 2012 to December 2019 were evaluated from the electronic health record of Aurora Health Care. Institutional review board approval was obtained. Statistics included descriptive (demographics) and categorical variables analyzed using chi-squared test. Continuous variables were analyzed using $t$ tests.

Results: In all, 4485 patients with hematologic malignancies across 17 oncology clinics were evaluated. Mean age was 68.3 years (range: 18-99), and the study population was predominantly White (89\%). Patients receiving chemotherapy, immunotherapy, and radiation therapy were more likely to be tested $(\mathrm{P}>0.01)$. The most common hematologic malignancy was chronic lymphocytic leukemia (21\%), followed by diffuse large B-cell lymphoma (18\%). A total of 645 (14\%) patients had a vitamin D test done within a 3-month window either before or after diagnosis, and 172 of 645 (27\%) had VDD. Patients with multiple myeloma (25\%) were the most likely to be tested for VDD, whereas individuals with primary myelofibrosis (7\%) were least likely. VDD was associated with an increased time to initial treatment for all combined hematologic malignancies.

Conclusion: Despite a reported prognostic value, vitamin D levels were infrequently assessed at diagnosis of hematologic malignancy. The role of VDD and treatment outcomes will be evaluated by stratifying hematologic malignancy tumor types and therapies.

\section{Universal COVID-19 Testing in Labor and Delivery Patients at Aurora BayCare Medical Center: Preliminary Results}

Jennifer K. Homa, Taylor A. Romdenne, Christina C. Renz, Kelly K. Malloy

\section{Advocate Aurora Research Institute; Aurora BayCare Medical Center}

Background: Pregnant women and their fetuses have been significantly impacted with increased risk of complications by emerging infections. Little is known regarding the impact of the novel SARS-CoV-2 infection (COVID-19) on delivery outcomes. Purpose: To evaluate rates of asymptomatic COVID-19 infection among laboring patients in northeast Wisconsin and any impact on delivery outcomes. 
Methods: A retrospective public health surveillance project was conducted as part of a universal COVID-19 testing policy at Aurora BayCare Medical Center (Green Bay, WI) with laboring patients from April 14, 2020, to July 31, 2020. Chart reviews were conducted to collect demographic data, COVID-19 results (mother and infant), and delivery outcomes (Apgar score, delivery method, gestational age, and arterial blood gas). Descriptive and frequency statistics were used to summarize the characteristics of the study population.

Results: Of the 487 singleton deliveries included, 9 mothers tested positive, 477 mothers tested negative, and 1 mother refused to be tested. Overall COVID-19 positive rate in northeast Wisconsin was $1.8 \%(\mathrm{n}=9)$. The asymptomatic COVID-19 positive rate was $1.6 \%$ $(n=8)$. Two mothers $(0.4 \%)$ were symptomatic; however, only 1 tested positive, yielding a symptomatic COVID-19-positive rate of $0.2 \%(n=1)$. None of the newborns in this sample tested positive for COVID-19. Notably, although only $9.4 \%$ of our sample were Hispanic women, $88.9 \%(n=8)$ of the COVID-19-positive cases were among Hispanic women.

Conclusion: Racial and ethnic disparities in COVID-19 cases have been observed, and these preliminary findings may help to support and guide local outreach efforts in northeast Wisconsin. Additional data are needed to monitor the impact of COVID-19 infection on pregnant women and their newborns.

\section{Encouraging Opioid Abstinence Through Contingency Management: A Pilot Study}

Elizabeth Wanninger, Mindy R. Waite, Rebecca Dizon-Ross, Ariel Zucker

Aurora Behavioral Health Services, Advocate Aurora Research Institute; University of Chicago Booth School of Business; Department of Agricultural \& Resource Economics, University of California, Berkeley

Background: Contingency management (CM) is an intervention that uses planned contingencies to change behavior. Typically, CM targets consequences to reward or punish behavior. CM has been tested across many behaviors, including substance use. Although $\mathrm{CM}$ has shown effectiveness for substance use populations, questions remain about the most efficient versus effective intervention designs. CM mobile applications have been developed to increase efficiency. However, effectiveness of automated CM mobile apps is not yet established.

Purpose: To test effectiveness of CM delivery via mobile app. This pilot tested proposed study methods and assessed whether opioid abstinence levels differed between treatment groups.

Methods: Patients were eligible if recently enrolled in partial hospitalization or intensive outpatient programs for an opioid use disorder at Aurora Health Care. Participants were randomly assigned to 1 of 3 groups. The Inputs group received differential reinforcement of alternative responses, including taking oral buprenorphine and attending substance use treatment. The Outcomes group received differential reinforcement of other behavior-completing opioidnegative saliva tests. The Combined group received both Inputs and Outcomes reinforcement. Activities were prompted and rewarded through the DynamiCare app (DynamiCare Health). All groups were provided an average of 13 tasks per month. Primary outcome was opioid-negative status on drug tests at 4-week intervals. Secondary outcomes included the number of successful tasks and substance use program completion rate.
Results: Drug screens were obtained at $59 \%$ of the 4-, 8-, and 12week interval markers. Respective rates of opioid-negative drug tests completed for weeks 4,8 , and 12 were $80 \%, 80 \%$, and $57 \%$ for Inputs; $83 \%, 100 \%$, and $100 \%$ for Outcomes; and $100 \%$, $75 \%$, and $60 \%$ for Combined. Inputs participants averaged 13.6 successful behaviors, Outcomes averaged 20.8, and Combined averaged 11.8. Completion rates for substance use treatment were $70 \%$ for Inputs, $75 \%$ for Outcomes, and $75 \%$ for Combined.

Conclusion: The pilot study showed success in enrolling patients and collecting outcomes data. However, opportunities for improvement were identified. Increased engagement in outcomes testing will require larger incentives. Also, due to low app engagement by a subset of participants across groups, practice opportunities should be added in the first week of enrollment. Providing participants earlier access to rewards may increase potential for maintaining opioid abstinence.

\section{Cardiac, Renal, and Liver Function in Neonates With Hypoxic Ischemic Encephalopathy Treated With Therapeutic Hypothermia}

Gospodin Stefanov, Timothy Colombo, Preetha Prazad, Ramona Donovan, Lorene Schweig

Neonatology, Advocate Children's Hospital; Rosalind Franklin University of Medicine and Science; Advocate Aurora Research Institute

Background: Therapeutic hypothermia $(\mathrm{TH})$ reduces the brain injury and negative multiorgan impact of perinatal asphyxia in neonates. TH is an established treatment for perinatal asphyxia, but there is a lack of research data to explain how TH modulates cardiac, renal, and hepatic dysfunction. Moreover, it is unclear if these functional changes can predict neonatal outcomes.

Purpose: To evaluate cardiac, renal, and liver function in neonates treated with $\mathrm{TH}$ and to determine whether various biochemical/ functional parameters are significant predictors of mortality.

Methods: A retrospective electronic medical record review of 47 neonates treated with TH because of perinatal asphyxia in a Level IV neonatal intensive care unit. All study procedures were approved by the local institutional review board. Subjects were divided into groups dependent on their: 1) gestational age at birth: late preterm or term; 2) size at birth: small or appropriate for gestational age; and 3) outcome: alive $(\mathrm{n}=40)$ or deceased $(\mathrm{n}=7)$. Data collected for analyses included cardiac, renal, and hepatic function parameters, concomitant medications/treatments, and perinatal factors. Oneway ANOVA and Pearson's correlation analyses were used to compare variables between the independent groups. Fisher's exact test was used for categorical variables.

Results: There was no significant difference in echocardiographic cardiac function parameters (ejection fraction, systolic function, end-diastolic left ventricular internal diameter [LVIDd]) between the alive and deceased groups $(\mathrm{P}>0.05)$. No significant correlation was found between ejection fraction, LVIDd, or systolic function and any of measured cardiac (troponin I, creatine kinase-MB), renal (blood urea nitrogen $[\mathrm{BUN}], \mathrm{BUN} /$ creatinine ratio, glomerular filtration rate [GFR]), or hepatic (alanine aminotransferase [ALT], aspartate aminotransferase [AST], alkaline phosphatase, lactic acid) biomarkers ( $P>0.05$ for all). Mean GFR and urine output were significantly lower and serum creatinine was significantly higher in the deceased group than the alive group at 24, 48, 72, and $96( \pm 4)$ hours after birth $(\mathrm{P}<0.05$ and $\mathrm{P}<0.005$, respectively). 
Mean serum BUN was not significantly different at any time point between the alive and deceased groups $(\mathrm{P}<0.05)$. Mean serum ALT, AST, and lactic acid were significantly higher in the deceased group than the alive group at 24 hours of life $(\mathrm{P}<0.05)$. No significant differences were found among the cardiac, renal, and liver function parameters between the gestational age or size at birth groups $(\mathrm{P}>0.05$ for all).

Conclusion: Cardiac, renal, and liver function parameters did not significantly differ based on gestational age or by weight for gestational age in neonates treated with TH. Markers of renal and hepatic function may be predictive of survival in neonates with perinatal asphyxia being treated with $\mathrm{TH}$.

Evaluation of Partial Thromboplastin Time Versus AntiXa Levels in Heparinized Patients With Left Ventricular Assist Device

\section{Bret Lentz, Jon Godden, Rachel Pedersen}

Pharmacy, Aurora St. Luke's Medical Center; Biostatistics, Aurora St. Luke's Medical Center

Background: Various studies have shown a discordance between activated partial thromboplastin time (aPTT) and anti-Xa levels in heparinized patients with a left ventricular assist device (LVAD). This discordance could lead to unwanted clinical outcomes if heparin therapy is not properly assessed in these high-risk patients. Patients with LVAD require precise monitoring to reduce the risk of both thrombus and hemorrhage. Aurora St. Luke's Medical Center (Milwaukee, WI) currently utilizes aPTT to routinely monitor heparinized patients. The goal of this retrospective review is to determine if aPTT and anti-Xa levels correlate and represent accurate heparin serum concentrations.

Purpose: To assess correlation of aPTT and anti-Xa levels in heparinized patients with LVAD.

Methods: We conducted a retrospective observational assessment of patients with LVAD requiring intravenous heparin who were monitored with aPTT and anti-Xa levels simultaneously. Serial paired levels of anti-Xa and aPTT were analyzed. Primary outcome was to assess the correlation between aPTT and anti-Xa based off hospital therapeutic heparin metrics. Categorical variables were described using frequency and percentages, and continuous variables were described using mean \pm standard deviation or median with interquartile range. A simple Kappa estimate was used to calculate agreement between anti-Xa and aPTT group levels. The project was determined to not constitute human subjects research and thus did not require institutional review board oversight.

Results: A total of 22 patients with 191 serial-paired anti-Xa and aPTT levels were included for analysis. Mean age at implant was $54.5 \pm 13.3$ years, $17(77.3 \%)$ were male, and $19(81.8 \%)$ implants were classified as destination therapy. Of the 191 levels, $42(22 \%)$ were indicated as subtherapeutic by both aPTT (ie, <45) and anti$\mathrm{Xa}(\mathrm{ie},<0.3)$. There were $82(43 \%)$ indicated as therapeutic by aPTT (ie, 45-70) but subtherapeutic by anti-Xa. Only 37 (19\%) were indicated as therapeutic by both aPTT and anti-Xa. Finally, 2 $(1 \%)$ were indicated as supratherapeutic by both aPTT and anti-Xa (Kappa estimate: $0.1,95 \%$ CI: 0.01-0.18).

Conclusion: Therapeutic groupings indicated by aPTT levels were in slight agreement when compared with anti-Xa. The most common disagreement group was where aPTT indicated a therapeutic range and anti-Xa indicated subtherapeutic. In our institution, this tended to overestimate the concentrations of heparin in the body.
Detrimental Oxygen: Oxygen Supplementation in Patients With ST-Segment Elevation Myocardial Infarction

\author{
Gabrielle Rubin, Namra Butt, Sorabh Kothari, Moustafa \\ Nagui Sabri
}

\section{Cardiology, Advocate Lutheran General Hospital; Cardiology, Advocate Christ Medical Center}

Background: There is evidence that supplemental oxygen administration in an acute myocardial infarction is associated with worse outcomes and larger infarct size.

Purpose: Currently, in the emergency room (ER), patients are being placed on oxygen supplementation regardless of their oxygen saturations. This quality improvement project aimed to reduce the number of patients with ST-segment elevation myocardial infarction (STEMI) and normoxia receiving supplemental oxygen in the ER by educating the ER staff about the harmful effects of oxygen in STEMI patients.

Methods: We retrospectively reviewed our acute STEMI population from February 1, 2020, to November 25, 2020, to assess how many of these patients were inappropriately placed on supplemental oxygen. We used a cut-off value of $\mathrm{Sp} 0_{2}$ greater than $90 \%$ as a threshold for inappropriate supplementation, in accordance with the published literature. We then provided education to all first point-of-care staff members on the appropriate use of supplemental oxygen and measured for changes in behavior. Our aim was to reduce inappropriate supplemental oxygen use by $50 \%$ in 3 months.

Results: Of the 126 total patients identified over the study period, $79 \%$ were placed on supplemental oxygen despite having an oxygen saturation greater than $90 \%$.

Conclusion: We identified a clear potential for harm with excessive use of supplemental oxygen for acute STEMI patients. Our goal is to obtain follow-up data at 3-month intervals to monitor for improvement, with plans to provide re-education if our goal of a $50 \%$ reduction in inappropriate supplemental oxygen use is not achieved.

\section{Evaluation of Rocuronium Dosing in Rapid Sequence Intubation Based on Ideal Versus Nonideal Body Weight in Obese Patients: A Prospective, Observational Study}

Amanda Lewandowski, Alaa Sulh, Michael Cirone, Mary Hormese, Marc McDowell

Pharmacy, Advocate Christ Medical Center; Pharmacy, Loyola University Medical Center; Emergency Medicine, Advocate Christ Medical Center; Pharmacy, Northwest Community Hospital

Background: Rapid sequence intubation (RSI) that provides adequate paralysis while reducing potential undersedation can be difficult in obese patients. Pharmacokinetic parameters play an important role in dosing of rocuronium, as it has low lipophilicity. Theoretically, rocuronium dosing should be based on ideal body weight, as accumulation does not occur in adipose tissue. In obese patients, no guideline-recommended dosing strategies exist for the use of rocuronium for RSI in the emergency department (ED), therefore dosing remains dependent on practitioner preference toward total body weight, ideal body weight, or adjusted body weight. A paucity of data has compared the use of rocuronium in obese patients based on different body weights. However, these 
studies took place in a surgical setting and not in the ED, therefore demonstrating the need for more data in an ED setting.

Purpose: To compare intubation conditions, duration of paralysis, and incidence of suboptimal sedation after intubation in obese patients requiring RSI with rocuronium in the ED.

Methods: This is a single-center, prospective, observational study. Subjects $\geq 18$ years of age with a total body weight $\geq 30 \%$ greater than ideal body weight or a body mass index of $\geq 30 \mathrm{~kg} / \mathrm{m}^{2}$ who presented to the ED requiring RSI with rocuronium were eligible for enrollment. Rocuronium was dosed according to physician/ pharmacist preference per current ED practice. For the calculation of rocuronium dose based on preferred weight, the pharmacist either utilized height to calculate ideal body weight or, when available, actual weight measurement obtained using either standard ED process or by reviewing prior documentation in the chart. After intubation, the physician completed a brief survey to assess intubation conditions utilizing a validated 9-point survey scoring system. The pharmacist noted height and weight used for the calculation of the dose, dose of rocuronium, time of medication administration, time of intubation, need for any repeat dose of paralytic, and time of muscle function recovery. Endpoints assessed included outcome of optimal intubation conditions, duration of paralysis, and incidence of suboptimal sedation (defined as the percentage of patients experiencing hypertension or tachycardia postintubation).

Results: Intubation was deemed "excellent" in $70.3 \%$ of subjects whose weight was calculated using standard ED process and in $67.9 \%$ of those with weight obtained from chart documentation; only the latter group received any intubation assessments of "poor" (6.7\%). Differences between groups when comparing noninferiority, first pass success, postintubation hypertension, and postintubation tachycardia were not statistically significant. For duration of paralysis, mean time to muscle recovery was 71 minutes (interquartile range: 57-96) for 40 subjects using total body weight and 43 minutes (40-60) in 33 subjects using ideal body weight $(\mathrm{P}<0.01)$.

Conclusion: Ideal body weight dosing of rocuronium is suggested to be noninferior to total body weight dosing in obese patients who require RSI in the ED. This sample size demonstrated similar efficacy of intubation conditions with shorter duration of paralysis between the two dosing-by-weight strategies. Follow-up superiority studies with a larger patient population are suggested to determine if there is a difference in optimal intubation conditions between dosing based on ideal body weight or total body weight.

\section{Rocuronium Versus Succinylcholine in the Traumatically Injured Brain: A Prospective Pilot Study}

Nadine Alwawi, Lauren Stambolic, Marc McDowell, Sabrin Jaber, Brook Walsh, Ellen C. Omi, Robert Mokszycki

Pharmacy, Advocate Christ Medical Center; Stanford University Medical Center; Little Company of Mary Hospital; Trauma Surgery, Advocate Christ Medical Center; University of Massachusetts

Background: Acute traumatic brain injury (TBI) often requires rapid sequence intubation (RSI) to protect patients' airways. RSI begins with pharmacologic induction, followed by paralysis with agents such as rocuronium or succinylcholine. These medications benefit from a rapid onset and a relatively short duration. Previous retrospective literature demonstrated a mortality benefit favoring rocuronium over succinylcholine.
Purpose: To assess mortality, among other outcomes, following RSI with rocuronium compared to succinylcholine for patients presenting to the emergency department (ED) with acute TBI.

Methods: This ongoing study identified patients for inclusion as of September 1, 2018. The study population consists of patients presenting to the ED with acute TBI who undergo RSI with either succinylcholine or rocuronium. Demographics recorded include age, sex, race, severity of TBI, and initial Glasgow Coma Scale (GCS). Exclusion criteria include: patient age of $<18$ years, surgical airway placed, pregnant at time of enrollment, cardiac arrest occurred prior to intubation, or intubation was attempted prior to arrival. Primary clinical outcome evaluated was incidence of inhospital mortality. Secondary outcomes measured both hospital and intensive care unit (ICU) length of stay (LOS). A sample size of 200 was calculated to detect a $20 \%$ difference in mortality.

Results: Of 77 patients evaluated in the study thus far, 60 were included for evaluation and 17 were excluded. Within the inclusion population, 42 patients were administered succinylcholine prior to intubation and 18 patients were administered rocuronium. Baseline demographics were comparable between both groups. Anticoagulant ( 5 vs $2 ; \mathrm{P}=0.9$ ) and antiplatelet ( 3 vs $3 ; \mathrm{P}=0.35$ ) use was reported in both groups. Prior to intubation, average GCS between the succinylcholine and rocuronium arms (6.5 vs 5.7, respectively; $\mathrm{P}=0.42$ ) did not show a significant difference. No statistical difference was detected in the incidence of in-hospital mortality (33.3\% vs $27.8 \%$; $\mathrm{P}=0.67)$, in-hospital LOS (13 vs 9.5 days; $\mathrm{P}=0.43$ ), or ICU LOS ( 7.4 vs 5.7 days; $\mathrm{P}=0.39$ ) between the two treatment groups.

Conclusion: Interim results from this ongoing study are inconclusive. These results prompt further investigation with additional patients enrolled.

\section{The Palliative Care Symptoms Assessment Tool and the Palliative Care Referral Criteria Index's Major Criteria Were Equally Effective at Increasing Palliative Care Referrals} Nora Badi, Alexander Schwank, Marianne Klumph, Kavita
Sharma

Hospice and Palliative Care Medicine, Aurora Sinai Medical Center; Aurora UW Medical Group; Center for Urban Population Health; Palliative Medicine, Aurora St. Luke's Medical Center

Background: There is growing evidence that early access to palliative care specialists can have a positive impact on quality of life through symptom management, reducing hospital admission and length of stay, improving overall patient care by addressing goals of care and health care directives, and even prolonging survival of patients with cancer. However, a main barrier to providing this care early and on time is the difficulty in identifying patients who could benefit from it.

Purpose: To compare the effectiveness of the Palliative Care Symptoms Assessment (PCSA) tool with the major and minor criteria of the Palliative Care Referral Criteria Index (PCRCI) in appropriately identifying and creating an effective method for referrals to palliative care services within the Vince Lombardi Cancer Clinic at Aurora Sinai Medical Center (Milwaukee, WI).

Methods: Patients undergoing transfusion at the Vince Lombardi Cancer Clinic for an oncology-related diagnosis who either filled out a PCSA or met the major or minor criteria of the PCRCI were 
identified via chart reviewed. Patients were referred to palliative care services based on a calculated score for the PCSA. Referral by PCRCI major criteria occurred if at least 1 of its criterions were met; the same standard applied to PCRCI minor criteria. Nonparametric methods were utilized for within-group and between-group differences. A P-value of 0.05 was considered statistically significant.

Results: Overall, 27 patients (38\% female) were included in the study sample, for which average age was $59 \pm 12$ years. The PCSA and PCRCI major criteria referred a similar percentage of patients $(89 \%$ vs $85 \%$; $\mathrm{P}=0.69)$. By contrast, the PCRCI minor criteria referred significantly fewer patients than the PCSA $(48 \%$ vs $89 \% ; \mathrm{P}=0.001$ ). An association between the screening tools was determined by the percentage of clients who were referred by both the PCSA and PCRCI major criteria $(n=22 \quad[82 \%]$; $\mathrm{P}=0.05)$ or both the PCSA and PCRCI minor criteria $(\mathrm{n}=13$ [48\%]; $\mathrm{P}=0.22$ ). Total PCSA scores between those referred vs not referred within PCSA, major criteria, and minor criteria were as followed: median (interquartile range) of 50 (24-79) vs $6(0-8)$ $(\mathrm{P}=0.01) ; 61(42-84)$ vs 21 (9-57) $(\mathrm{P}=0.02)$; and 42 (17-83) vs $39(3-74)(\mathrm{P}=0.38)$.

Conclusion: The PCSA and the PCRCI's major criteria were equally effective at referring patients, whereas the PCRCI's minor criteria performed poorly. Future research should focus on whether the major criteria would still be as effective with the exclusion of the severe symptoms criteria, as severe symptoms are determined by the PCSA.

\section{KALMED: Ketamine for Acute Agitation Management in the Emergency Department}

Lauren Stambolic, Marc McDowell, Dharati Desai, Jaxson Burkins, Nadine Lomotan, Tasneem Ahmed, Stephany Nuñez Cruz

Pharmacy, Advocate Christ Medical Center; Emergency Department, Advocate Christ Medical Center

Background: Acute agitation is common in the emergency department (ED). It is crucial to efficiently relieve agitation in these patients for the safety of the patients and the health care team. Numerous studies conducted in the prehospital setting and in the ED have found ketamine to be an effective agent with more rapid onset of action compared with benzodiazepines or antipsychotics for initial control of acute agitation. To date, there are no data comparing reduced-dose ( $\sim 2 \mathrm{mg} / \mathrm{kg})$ vs standard-dose $(4-5 \mathrm{mg} / \mathrm{kg})$ intramuscular ketamine for acute agitation.

Purpose: To assess the effectiveness and safety of intramuscular ketamine of $<2.5 \mathrm{mg} / \mathrm{kg}$ vs $>2.5 \mathrm{mg} / \mathrm{kg}$ for acute agitation.

Methods: This was a single-center, retrospective, 2-arm study cohort. Patients who received intramuscular ketamine for acute agitation in the ED were included. Exclusion criteria included patients under 18 years old or administration of a concomitant sedative agent during ketamine administration. Primary outcome was resolution of agitation at 15 minutes ( \pm 10 minutes) after ketamine administration. Resolution of agitation was defined as documentation from a health care provider, lack of administration of another sedative agent within 30 minutes from ketamine administration, or the ability to complete necessary procedures. Secondary outcomes included use of intramuscular or intravenous rescue medications within 30 minutes after ketamine administration, adverse events, and time to medical clearance.
Results: There was no statistically significant difference in resolution of agitation between the low-dose and high-dose intramuscular ketamine groups $(91.4 \%$ vs $100 \%$, respectively; $\mathrm{P}=0.54$ ). There was a nonsignificant increase in intubation in the low-dose group compared to the high-dose group $(11.4 \%$ vs $6.3 \% ; \mathrm{P}=1.00$ ); however, the majority of intubations ( 4 of 5 ) were unrelated to ketamine administration.

Conclusion: Expanding this project's time frame for data collection is needed to yield sufficient power and better inform future prospective study design.

\section{Access to Targeted Online Educational Resources Before and in the Midst of a Pandemic}

Deborah Simpson, Kathryn Denson, Amanda Szymkowski, Debra Mooney, Steven Denson, Edmund H. Duthie, Jr., Michael L. Malone

Family Medicine and Academic Affairs, Aurora Sinai Medical Center; Geriatrics, Medical College of Wisconsin; Geriatrics, Aurora Sinai Medical Center; Aurora Senior Services, Aurora UW Medical Group

Background: In 2020 medical education shifted to virtual settings driven by pandemic-related precautions. New venues for education were created. Long-standing virtual forums, such as a monthly 1-hour most difficult case conference (MDCC) series for geriatric fellows across the country, continued without disruption - transitioning from audio-only to include video. As an existing national forum, the Advocate Aurora Health (AAH)-sponsored MDCC is uniquely positioned to evaluate if access rates for educator-recommended virtual resources changed from prepandemic to midpandemic. Understanding rate change during times of stress is essential for educators to knowledgeably adapt instruction.

Purpose: To determine if access rates changed for educatoridentified virtual resources referenced during a pandemic.

Methods: Geriatric Fast Facts (GFF) is a collaboration between $\mathrm{AAH}$ and the Medical College of Wisconsin that provides a virtual resource for teachers/learners and contains peer-reviewed, evidence-based summaries on topics essential to older adult care via a searchable website (www.geriatricfastfacts.com). A GFF specific to each MDCC monthly case was provided via a link to conference participants. Google Analytics data were used to determine if site traffic for the MDCC-targeted GFF differed pre- vs midpandemic. Average daily access rate 12 days prior to each MDCC session was calculated as a control and compared to the access rates on the MDCC day. Site traffic data are typically stable day-to-day, and there is no site registration.

Results: On average, MDCC attendance increased by $45 \%$ from prepandemic (13 sessions held during May 26, 2019-February 19, 2020) to midpandemic (13 sessions held during April 15, 2020-November 18, 2020). Access rates to MDCC-targeted GFF remained constant from pre- to midpandemic given MDCC participation increase. MDCC-targeted GFF site visits averaged 6.06 pre- and 10.09 midpandemic, a 44\% increase (average increase of 4.9 per MDCC session). Individuals stayed on the GFF website, visiting 16.54 other pages pre- and 25.19 midpandemic, a $34 \%$ increase (average increase of 8.7 per MDCC session).

Conclusion: MDCC-linked GFF access and other page visits remained constant during the pandemic. Educators can be confident that their efforts to identify relevant, synoptic web resources are still accessed even in challenging times. 


\section{Providing AC2Tionable Feedback Improves Resident and Fellow Satisfaction}

Deborah Simpson, Jacob L. Bidwell, Tricia La Fratta, Will Lehmann, David Hamel, Nicole P. Salvo, William MacDonald, Aboud Affi, M. Eyman Mortada, Colleen Nichols

Family Medicine and Academic Affairs, Aurora Sinai Medical Center; Aurora Graduate Medical Education; Aurora UW Medical Group; Family Medicine, Aurora St. Luke's Medical Center; Internal Medicine, Aurora Sinai Medical Center; Obstetrics and Gynecology, Aurora Sinai Medical Center; Radiology, Aurora St. Luke's Medical Center; Gastroenterology, Aurora Sinai Medical Center; Aurora Cardiovascular Services, Aurora St. Luke's Medical Center

Background: Feedback is an essential component of the learning process and is a required element for graduate medical education program accreditation. Yet, it is often among the lowest-rated items on education/teaching evaluations, is stressful for learners/teachers alike, and is not actionable. To be actionable, feedback must include current performance (omission/commission, deficient/ error), the desired goal performance (the gap), and a stepwise approach to bridging that performance gap with strategies and resources. Unfortunately, one or more of these elements is often omitted, rendering most feedback unactionable.

Purpose: To educate learners and teachers to seek/provide actionable feedback and determine if residents' and fellows' feedback ratings improve.

Methods: Over a 2-year period, faculty and trainees in Accreditation Council on Graduate Medical Education (ACGME)-approved residency/fellowship programs in our sponsoring institution were invited to attend a 45-minute actionable feedback session. The session was typically incorporated into an established training program venue. The core elements of actionable feedback were highlighted from both a faculty and learner perspective using AC2T model: "A" = Ask for feedback/Answer focused on specific performance gap; "C" = Clarify if any actionable feedback elements were omitted; "C" = Consider what steps you will take/Coach to support learning and growth; " $T "=$ say Thanks, as feedback is a dynamic relationship. Sessions included feedback vignettes framed around specialtyspecific milestones to provide practice opportunities. Participant pairs were given 90 seconds to complete the feedback interaction, followed by a debriefing. ACGME-administered survey results over 4 years were examined for the 2 years prior to $\mathrm{AC} 2 \mathrm{~T}$ and the 2 years of AC2T implementation.

Results: In each of the 2 years prior to AC2T implementation, our ACGME mean rating on the item "satisfied with feedback" was significantly below the national mean by 0.4 (5-point scale from "extremely satisfied" to "not at all satisfied"). Response rate was $>98 \%$ : 139 of 141 for 2017 ; 163 of 167 for 2018). After AC2T education in year 1 , feedback item ratings improved to 0.20 below national mean (response rate: $99 \%$ [168 of 170]); in year 2, ratings reached the national mean (response rate: $85 \%$ during pandemic [152 of 179]).

Conclusion: Focusing learner and faculty development on key elements of actionable feedback is associated with dramatic improvement in feedback ratings.
Personal Protective Equipment Compliance: A Quality Improvement Initiative

Hilary Brown, Lizabeth Copre, Catherine Larson, Stephanie Spacone-Perez, Laura Albanese, Sandra Weitzner, Mitali Shah, Varsha P. Gharpure

\section{Chicago Medical School; Advocate Children's Hospital}

Background: Provider and patient safety is dependent on accurate donning and doffing of personal protective equipment (PPE). Studies prior to the COVID-19 pandemic have reported poor adherence to PPE use, with higher risk of contamination during doffing. Prepandemic audits at the pediatric general ward and intensive care unit of Advocate Children's Hospital (Park Ridge, IL) showed varying compliance each month (88\%-100\%) with donning. Doffing historically was never monitored. The COVID-19 pandemic has heightened the importance of donning and doffing while making the process more complex due to need for additional equipment and reuse of supplies.

Purpose: To identify and ameliorate modifiable individual and system factors through process standardization and education to achieve 100\% compliance with PPE use.

Methods: The local institutional review board approved this multidisciplinary quality improvement initiative. We used an anonymous online survey (REDCap) and bedside audits to assess baseline compliance and opportunities for intervention.

Results: 86 providers ( 27 physicians/advance practice providers, 38 nurses, 20 respiratory therapist, and 1 ancillary services) returned the survey. Electronic communication from leadership $(63 \%)$ and an online COVID-19 toolkit (56\%) were reported to be major sources of information, while $13 \%$ had participated in in-situ simulation. Only 20\% reported to be very confident of their own donning/doffing skills, while $29 \%$ were somewhat confident. About $30 \%$ of team members self-reported errors/contamination during donning or doffing; reasons were identified as lack of knowledge of the current processes due to frequent changes in recommendations (74\%), lack of supplies consolidated in one location (89\%), distraction from phone calls/conversation (88\%), patient deterioration (66\%), and PPE fatigue (33\%). Baseline audit revealed use of appropriate PPE by most providers, but the process was often completed out of order or incorrectly.

Conclusion: We identified a need for additional education and readily available supplies. Videos showing proper donning and doffing technique, in-person validation of said technique, and justin-time review for team members are educational interventions to be implemented. Custom-designed, dedicated PPE carts are placed at the bedside for short-term storage and easy access to equipment. A postintervention survey and audit will be completed to assess the effectiveness of these interventions and identify future opportunities.

\section{Evaluating the Pediatric Transitional Care Management Visit at Aurora Sinai Family Care Center}

Mary M. Dunkin, Fabiana Kotovicz, Alonzo Jalan, Jessica J. F. Kram

Family Medicine, Aurora St. Luke's Medical Center; Aurora UW Medical Group; Center for Urban Population Health

Background: The U.S. Centers for Medicare \& Medicaid Services offers payment to outpatient primary care facilities to provide 
transitional care management (TCM) visits. The visit serves as a handoff of patients from the acute inpatient setting to the outpatient ambulatory environment, allowing succinct follow-up on medical management. However, creating a thorough workflow and protocol to establish TCM visits can be challenging, especially when patients are mostly discharged from hospital facilities that are not a part of one's health system.

Purpose: To assess the current pediatric TCM workflow at an academic primary care clinic, as well as missed opportunities for pediatric hospital discharge follow-up and appropriate TCM reimbursement.

Methods: The current pediatric TCM workflow was obtained via informal interviews with care-coordinating staff members of Aurora Family Care Center (Milwaukee, WI). A retrospective chart review was performed for clinic patients, 3 days-18 years of age, who were hospitalized during January-December 2019 and who were discharged from any community hospital to outpatient follow-up at Aurora Family Care Center. It was determined whether TCM visits were performed following their hospital discharge. Basic descriptive statistics and Mann-Whitney tests for continuous analyses were used as appropriate. For outpatient visits not meeting TCM criteria for billing, loss of reimbursement was calculated per patient's medical complexity at the time of their hospital discharge. Results: Of the 46 patients who met inclusion criteria, mean age was 7.3 years, mean hospital stay was 4.7 days, and $60.9 \%$ were male. The two most common discharge diagnoses were respiratory $(24 \%)$ and surgical (24\%). Overall, 22\% $(n=10)$ of pediatric patients received a follow-up visit after discharge. However, only $50 \%(n=5)$ of the hospital discharge visits met criteria for TCM billing. There were no statistically significant differences in whether a TCM visit was performed based on median length of stay $(2.5$ vs 3 days; $\mathrm{P}=0.82)$. However, there was a statistically significant difference based on age ( 0.2 vs 7.4 years; $\mathrm{P}=0.01)$. Reimbursement for those who met TCM criteria was $\$ 963.44$. The total estimated potential TCM reimbursement for patients not meeting criteria and who did not have a hospital follow-up was $\$ 7805.19$.

Conclusion: Team-based TCM services are designed to allow appropriate medical follow-up for recently discharged patients. Our primary care clinics have room to implement a more standardized and effective pediatric TCM process.

\section{Assessing Comfortability and Knowledge of LGBTQ Health in Family Medicine Residency Program}

\section{Steven Wipijewski, Jessica O’Brien}

\section{Family Medicine, Aurora St. Luke’s Medical Center}

Background: The LGBTQ community experiences health disparities, including higher mental health concerns and lower likelihood to receive recommended preventive care. A contributing factor to these disparities is a lack of physician comfort and education on LGBTQ health.

Purpose: To assess primary care residents' and attendings' medical knowledge and comfort of LGBTQ health issues before and after formal education.

Methods: A pretest survey was emailed to residents and faculty at the Aurora St. Luke's Medical Center (Milwaukee, WI) family medicine residency program. Questions included self-reported knowledge and comfort of taking care of LGBTQ patients, terminology, Centers for Disease Control and Prevention and U.S. Preventive Services Task Force screening guidelines, and gender- affirming treatment options. After completion of the pretest survey, two 15-minute lectures were provided during resident-faculty meetings. After completion of the second lecture, a posttest survey was emailed to residents and faculty.

Results: Pretest $(n=34)$ and posttest $(n=20)$ responses were compared using chi-squared tests and Fisher's exact test for categorical variables and $t$ tests for continuous variables. While respondents reported improvement in knowledge (43.4\% pretest vs $53.1 \%$ posttest) and comfort (52.0\% pretest vs $62.0 \%$ posttest) after the lecture series, results were not statistically significant ( $\mathrm{P}=0.161$ and $\mathrm{P}=0.191$, respectively). There were no statistically significant differences between pretest and posttest scores on LGBTQ health questions.

Conclusion: While self-reported knowledge and comfort scores improved after receiving lectures on LGBTQ health, results were not statistically significant. Interpretation of the data was limited due to fewer respondents on the posttest as well as having different respondents when comparing pretest and posttest identifiers. It is also unclear what percentage of residents and attendings were able to attend either or both lecture series and whether more or longer educational sessions would have increased medical knowledge and comfort further. Addressing barriers to physician attendance and completion of surveys could improve the study.

\section{Gabapentin for Prophylaxis of Alcohol Withdrawal}

\section{Caroline Kruszecki, Tyler Cooper, Poorvi Shah}

Pharmacy, Advocate Christ Medical Center; Internal Medicine, Advocate Christ Medical Center

Background: Limited information is available on the use of pharmacological agents for alcohol withdrawal prophylaxis. Current literature for benzodiazepines has been reported; however, the role of gabapentin as a prophylactic agent has yet to be determined.

Purpose: To evaluate the efficacy of gabapentin to prevent disease progression in patients at high risk of developing severe alcohol withdrawal.

Methods: This retrospective cohort study was approved by the institutional review board and used electronic medical records to identify patients who had Clinical Institute Withdrawal Assessment (CIWA) scores monitored during inpatient stay. Patients were included if their initial CIWA scores were $<8$ on admission, indicating that they were not experiencing active withdrawal symptoms. Patients who either experienced acute alcohol withdrawal symptoms on admission as defined by a CIWA score of $>8$ or were admitted for primary treatment of alcohol withdrawal or dependence were excluded. The intervention group consisted of patients who received gabapentin prior to a reported CIWA score of $>8$, while the control group consisted of those who did not. The following baseline characteristics were collected: age, sex, weight, serum creatinine, liver function tests, and history of alcohol withdrawal seizures or delirium tremens. Primary objective of this study was to compare the number of asymptomatic patients who progressed to severe alcohol withdrawal (defined as 2 CIWA scores of $>15$ within an 8-hour time period) when given gabapentin prophylaxis to those who were not given gabapentin prophylaxis. Secondary endpoints included medication use and duration (benzodiazepines, antipsychotics, phenobarbital, propofol, and alpha-2 agonists), CIWA scores, need for intensive care unit (ICU) admission, 
incidence of mechanical ventilation, ICU and hospital length of stay, rates of complications, and select adverse effects.

Results: There were no differences in progression to severe withdrawal, hospital length of stay, need for rescue medications, benzodiazepine requirements, or adverse effects between patients who received gabapentin for alcohol withdrawal prophylaxis and those who did not. Median ICU length of stay was shorter among patients who received gabapentin, with $300 \mathrm{mg}$ every 8 hours the most commonly observed gabapentin dosing regimen.

Conclusion: Larger prospective studies evaluating gabapentin prophylaxis in high-risk patients are warranted. The lack of observed differences in our study may be attributed to the small sample size and the combination of low- and high-risk patients.

\section{Demographics, Treatment, and Survival for Patients Diagnosed With Pancreatic Cancer, 2010-2016}

\section{Maharaj Singh, Michelle Simpson}

\section{Advocate Aurora Research Institute; Ed Howe Center for Research, Aurora Sinai Medical Center}

Background: Incidence of and deaths caused by pancreatic cancer have increased over recent years despite improvements in mortality rate in other cancer types. Pancreatic cancer is a predominantly asymptomatic cancer, very difficult to detect, with diagnosis late in the disease. It is among the most lethal cancers.

Purpose: To describe the demographics among those who were diagnosed with pancreatic cancer, evaluate incidence, mortality, overall survival, and percent survival after 1, 2, and 5 years for patients diagnosed with pancreatic cancer.

Methods: Data from the National Cancer Database for 2010-2016 was used for this study. We identified 384,753 patients diagnosed with pancreatic cancer. In this study population, patients with missing mortality (dead, alive) were excluded from the study sample. Descriptive statistics and Cox regression were used to analyze the data.

Results: Overall, the study cohort was $49 \%$ female, $79 \%$ nonHispanic White (NHW), 12\% non-Hispanic Black (NHB), 5\% Hispanic, and 3\% defined as "Other" race (Asians and Pacific Islanders). Cox proportional hazard regression analysis showed that female (hazard ratio: $0.95,95 \%$ CI: $0.94-0.96$ ) vs male sex, Hispanic $(0.96,0.95-0.97)$, NHB $(0.94,0.93-0.95)$, and Other $(0.88,0.87-0.89)$ vs NHW race/ethnicity, lower-medium $(0.96$, 0.95-0.97), medium (0.94, 0.93-0.95), and high (0.88, 0.87-0.89) vs low patient income category, residence in metro $(0.99,0.98-$ 1.00) vs urban areas, living in the U.S. Eastern (0.96, 0.95-0.96) and Western $(0.99,0.98-1.00)$ vs Central region, and having government $(0.90,0.88-0.92)$ or private $(0.84,0.82-0.59)$ vs no insurance all had lower hazards for mortality. Similarly, patients who received treatment such as surgery $(0.29,0.29-0.30)$, radiation (0.85, 0.84-0.86), chemotherapy $(0.69,0.69-0.70)$, immunotherapy $(0.76,0.72-0.81)$, or hormonal therapy $(0.50,0.47-0.54)$ had lower hazards for mortality, as did patients with higher Charlson-Dayo score ( 1 vs $0: 1.13,1.12-1.14 ; 2$ vs $0: 1.27,1.25-1.29$; $\geq 3$ vs 0 : $1.48,1.14-1.52)$. Also, 1-year increment in age increased risk of dying by $1 \%-2 \%(1.01,1.01-1.02)$.

Conclusion: Female, Hispanic, Asian and Pacific Islander, and younger patients had better survival. Patients with insurance (government or private) and patients who underwent treatment had higher survival probability. Nationally, 5-year survival has improved from $6 \%$ in 2004 to $13 \%$ in 2014 .

\section{Review of Abametapir as a New Treatment for Head Lice*}

Alexander D. Woods, Caroline L. Porter, Steven R. Feldman

Center for Dermatology Research, Wake Forest School of Medicine

Background: Head lice, or Pediculus humanus capitis, is a common yet difficult to treat ectoparasite. It has traditionally been treated with over-the-counter synergized pyrethrin or pyrethroids (permethrin); however, widespread resistance has led to the need for novel pediculicides, such as abametapir $0.74 \%$ (Xeglyze).

Purpose: To review the pharmacology, safety, efficacy, and clinical importance of abametapir.

Methods: A systematic review of the MEDLINE and Embase databases was conducted for the terms "abametapir," "Xeglyze," or "Ha44." All relevant articles prior to December 2020 were included.

Results: Abametapir works through chelating heavy metal ions and inhibiting metalloproteinases critical to louse ova development, hatching, and adult survival. A phase 2 trial using an ex-vivo approach validated the direct ovicidal activity of abametapir, inhibiting $100 \%$ of treated louse eggs from hatching compared to $64 \%$ in the vehicle-treated group. With comparison to untreated controls, the absolute hatch rate reduction was $92.2 \%$ in abametapir-treated and $42.3 \%$ in vehicle-treated louse eggs $(\mathrm{P}<0.001)$. Two identical phase 3 clinical trials conducted in 704 patients with head lice infestations showed subjects treated with a single 10-minute application of abametapir had a significantly greater proportion of treatment success (ie, lice-free through 14 days) compared to vehicle-treated subjects with $81.1 \%$ success vs $50.9 \%(\mathrm{P}=0.001)$ and $81.8 \%$ vs $47.2 \%(\mathrm{P}<0.001)$ in each respective trial. Abametapir was well tolerated, with mild scalp erythema, rash, skin burning sensation, and contact dermatitis being the most commonly experienced adverse effects.

Conclusion: Abametapir $0.74 \%$ was recently approved by the U.S. Food and Drug Administration for single-application treatment for head lice in patients 6 months of age and older. Its direct ovicidal and lousicidal activity is effective in treating head lice infestations with one application. In the face of growing resistance to current pediculicides, abametapir offers a safe and effective new treatment.

*Data republished from Woods AD, Porter CL, Feldman SR. Abametapir for the treatment of head lice: a drug review. Ann Pharmacother. 2021 Jun 22 [Epub ahead of print]; with permission from SAGE Publishing. 